\title{
A New Approach for Designing Gear Profiles using Closed Complex Equations
}

\author{
Bálint Laczik $^{1}$, Peter Zentay ${ }^{2}$, Richárd Horváth ${ }^{2}$ \\ ${ }^{1}$ Department of Manufacturing Science and Technology, Budapest University of \\ Technology and Economics, Egry József u. 1, H-1111 Budapest, Hungary, \\ laczik@goliat.eik.bme.hu \\ ${ }^{2}$ Óbuda University Donát Bánki Faculty of Mechanical and Safety Engineering, \\ Népszínház u. 8, H-1081 Budapest, Hungary, zentay@actel.hu, \\ horvath.richard@bgk.uni-obuda.hu
}

\begin{abstract}
The literature solely uses the homogeneous transformation matrix method for solving the problems of rotations and translations, for gear contact problems. In this paper a different approach is introduced, using complex algebra for designing the profiles of gear teeth. We intend to present the general theory and technique of designing the generating gear rack and the meshing counter profile of arbitrary profile gears. For the illustration of the practical use of his method we shall present the design steps of a gear, with a cosine profile, a general involute gear and the profile of the lobes of a Root blower pump. These examples will properly illustrate the applicability of the presented design process.
\end{abstract}

Keywords: gear profile; meshing theory; envelope; complex function

\section{Introduction}

One of the important machine elements, from the beginning of the technical era, is the gear. It has been used since the dawn of time in ancient Persia, Greece and the Roman age, usually for operating water pumps. These gear designs were not very efficient, mostly using the pin and crown gearing principle [1].

The first general application of the analytical tooth profile can be related to the works of L. Litvin [2]. His homogeneous matrix transformation method became increasingly popular from the time that the calculations could be performed by computers. New results for the classical problems were made possible with the help of computer aided mathematical deductions. The paper describes the representation of the gear contact principles using a complex algebraic method [2, $3,4]$. The solution of the equations is used for the generation of the gear profile, the contact line and also the generating gear rack tool profile. The practical use of 
the presented method is illustrated by three examples generating different gear geometries, such as, a cosine profile gear similar to a gear introduced in paper [5], a general involute gear and the profile of the lobes of a Roots blower pump [6,7]. The described method is manufacturing friendly, because the gear tooth profile is calculated from the gear rack, which is also the generating tool. By introducing a design method that gives the exact profile of the tool the gear production method is defined instantly and the manufacture of the gear is a straightforward process.

\section{The Method of Gear Profile Generation}

For the general method we follow the theory according to [8]. The straight line $\mathrm{g}_{1}$ of the rack (see: Fig. 1) is rolling without slipping on the rolling curve $g_{2}$ of the gear with radius $r$. The tooth element of the rack (1) is enveloping the tooth of the gear (2) (See Fig. 1 and also Fig. 2).

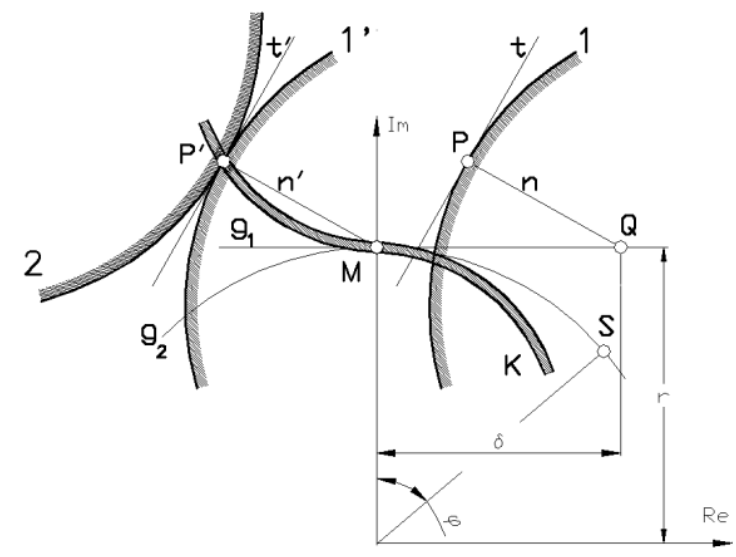

Figure 1

Geometric relation of conjugated profiles 


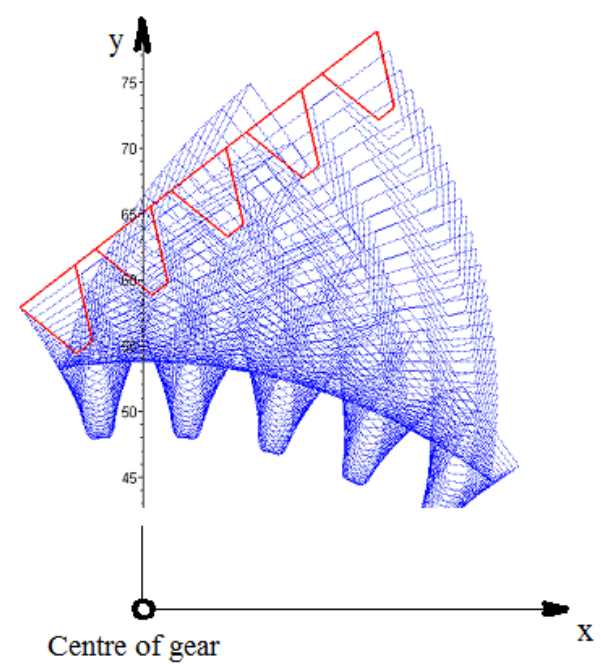

Figure 2

Generating the involute gear with the rack

The non-slip rolling of the profile of the rack (its straight line) on the centrode of the gear can be constructed from the following two motion elements (see: Fig. 3a to $3 \mathrm{c})$ :

I. The first motion is: the rack moves from the starting position 1 with the distance of $\delta=r \cdot \varphi$ in the direction of the real axis (position 1').

II. The second motion when the rack moves by a rotation around the origin with the angle $\varphi$ to the position 1". (Assuming plain rolling the gear centrode $\mathrm{g}_{2}$ is the arc length $M S=r \cdot \varphi$ and the length of the rolling straight line $\mathrm{g}_{1}$ is $T M=\delta$. From the figure the following equation can be obtained: $M S=T M$. (See: Fig. 3a).

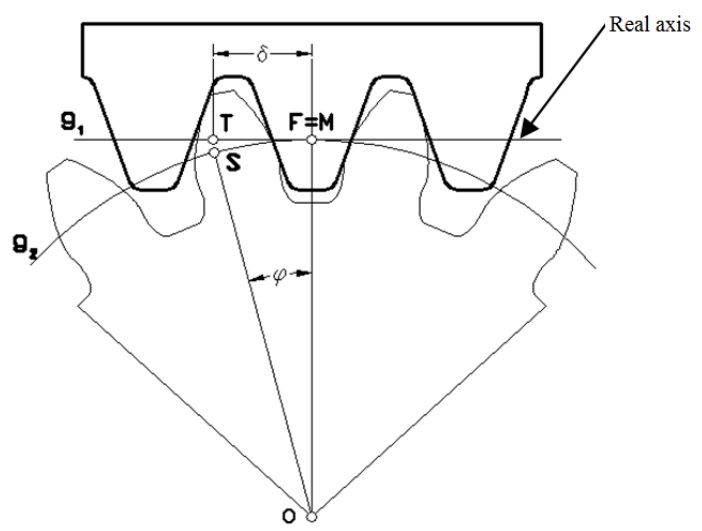

a) Starting position of the Rack 


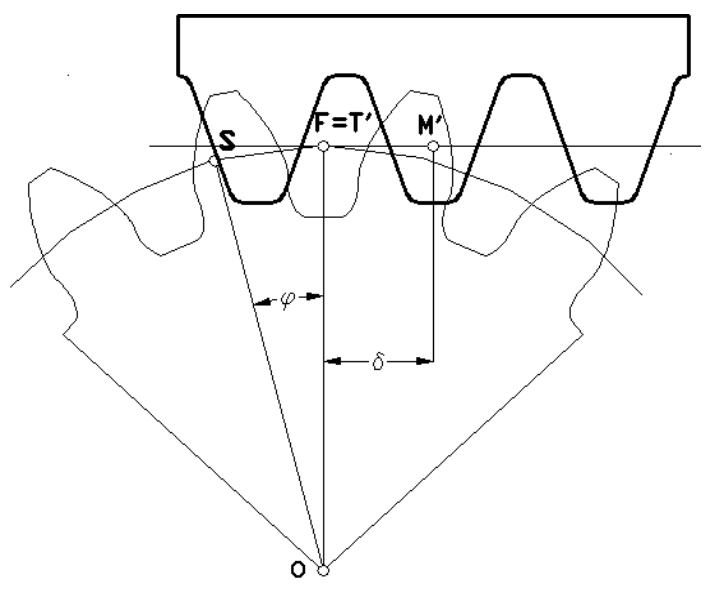

b) $1^{\text {st }}$ motion element of rolling

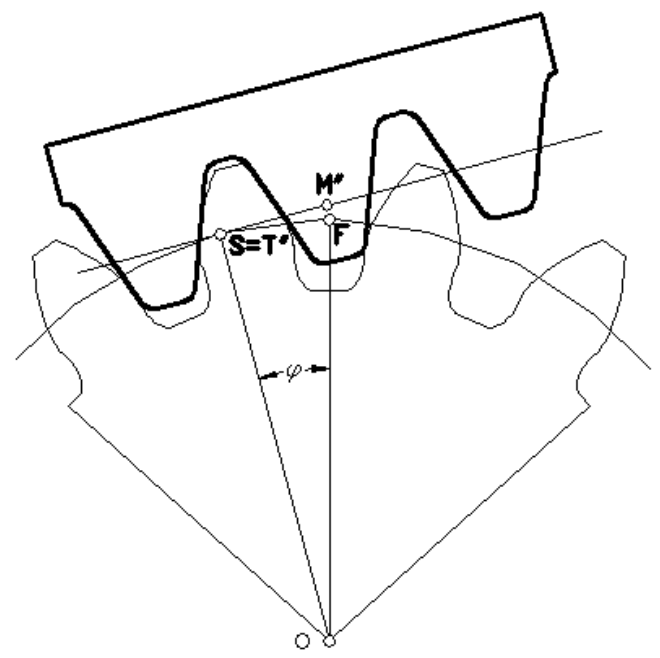

c) $2^{\text {nd }}$ motion element of rolling

Figure 3

The motion elements of gear generation

Fig. 3a) illustrates the starting position of the rack, Fig. 3b) the $1^{\text {st }}$ and Fig. 3c) the $2^{\text {nd }}$ motion element of the rolling. With the use of the motion elements (I and II) the shape enveloped by the motion of the rack and the gear profile meshing with it can be determined in a general way. Let the rack profile be given with a complex function with the parameter $u$, according to the following equation:

$z=z_{1}(u)+I \cdot z_{2}(u)$ 
The complex transformation formula of the motion elements I -II is given by the following formula:

$$
w=\left(z_{1}(u)+I \cdot z_{2}(u)+r \cdot \varphi\right) \cdot e^{(I \cdot \varphi)}
$$

where $r$ is the radius of the pitch curve, and $z_{1}$ and $z_{2}$ are the real and imaginary parts of the rack profile function.

During the roll the following differential equation is valid for the border form (2) (the gear teeth) generated by the rack given by equation (1). The real and the imaginary parts of the function are $R(w)$ and $I(w)$. During the roll the following differential equation is valid for the border form (2) (the gear teeth) generated by the rack given by equation (1). The real and the imaginary parts of the function are $R(w)$ and $I(w)$.

$$
\tan (\alpha)=\frac{\frac{\partial}{\partial \varphi} I(w(u, \varphi))}{\frac{\partial}{\partial \varphi} \operatorname{Re}(w(u, \varphi))}=\frac{\frac{\partial}{\partial u} I(w(u, \varphi))}{\frac{\partial}{\partial u} \operatorname{Re}(w(u, \varphi))}
$$

By expanding the differential equation (3) the following formula is gained:

$$
\begin{aligned}
& \frac{\left(\frac{d}{d u} z_{2}(u)\right) \cdot \cos (\varphi)+\left(\frac{d}{d u} z_{1}(u)\right) \cdot \sin (\varphi)}{\left(\frac{d}{d u} z_{1}(u)\right) \cdot \cos (\varphi)-\left(\frac{d}{d u} z_{2}(u)\right) \cdot \sin (\varphi)}- \\
& -\frac{-z_{2}(u) \cdot \sin (\varphi)+r \cdot \sin (\varphi)+\cos (\varphi) \cdot z_{1}(u)+\cos (\varphi) \cdot r \cdot \varphi}{r \cdot \cos (\varphi)-\sin (\varphi) z_{1}(u)-\sin (\varphi) \cdot r \cdot \varphi-z_{2}(u) \cdot \cos (\varphi)}=0
\end{aligned}
$$

An analytic (closed form) solution of the differential equation (4) can be obtained for the variable $\varphi$ :

$$
\varphi=\frac{-\left(\frac{d}{d u} z_{2}(u)\right) \cdot r+\left(\frac{d}{d u} z_{2}(u)\right) \cdot z_{2}(u)+z_{1}(u) \cdot\left(\frac{d}{d u} z_{1}(u)\right)}{r \cdot\left(\frac{d}{d u} z_{1}(u)\right)}
$$

The expression can be further simplified using the following substitutions:

$$
z_{1}(u)=F_{1}, z_{2}(u)=F_{2}, \frac{d}{d u} z_{1}(u)=f_{1}, \frac{d}{d u} z_{2}(u)=f_{2}
$$

The variable $\varphi$ can be expressed with the formula of the rack (1), and its derivatives according to $\mathrm{u}$. In this way a very simple formula is obtained for the variable $\varphi$ :

$$
\varphi=-\frac{-f_{2} \cdot r+f_{2} \cdot F_{2}+F_{1} \cdot f_{1}}{r \cdot f_{1}}
$$


The gear tooth profile is expressed as a complex equation which is dependent on the parameter $u$, in this way by substituting the variable $\varphi$ into the two-parameter equation the solution can be obtained in a closed form:

$$
K=\left(F_{1}+F_{2} \cdot I-\frac{-f_{2} \cdot r+f_{2} \cdot F_{2}+F_{1} \cdot f}{f_{1}}\right) \cdot e^{\left(-\frac{I \cdot\left(-f_{2} \cdot r+f_{2} \cdot F_{2}+F_{1} \cdot f\right)}{r \cdot f_{1}}\right)}
$$

To obtain a proof of the line of action the theorem of Willis is used. The equation of the profile tangent at the point given by the parameter $u$ - with complex coordinates $z_{l}(u)+I \cdot z_{2}(u)$ - for the line of action is:

$$
t=z_{1}(u)+I \cdot z_{2}(u)+\lambda \cdot\left(\left(\frac{d}{d u} z_{1}(u)\right)+I \cdot\left(\frac{d}{d u} z_{2}(u)\right)\right)
$$

The equation of the normal of the profile at the same point is:

$$
k=z_{1}(u)+I \cdot z_{2}(u)+\lambda \cdot\left(\left(\frac{d}{d u} z_{2}(u)\right)-I \cdot\left(\frac{d}{d u} z_{1}(u)\right)\right)
$$

According to the theorem of Willis the profile normal crosses the point with the following coordinates $0+I \cdot r$. Using this theorem the equation of connection can be formulated in the following way:

$$
z_{2}(u)-\lambda \cdot\left(\frac{d}{d u} z_{1}(u)\right)=r
$$

The connection can be expressed by solving equation (11) according to $\lambda$ :

$$
\lambda=\frac{z_{2}(u)-r}{\frac{d}{d u} z_{1}(u)}
$$

The next step is to substitute the value of $\lambda$ into the real part of equation (10):

$$
\delta=z_{1}(u)-\frac{\left(z_{2}(u)+r\right) \cdot\left(\frac{d}{d u} z_{2}(u)\right)}{\frac{d}{d u} z_{1}(u)}
$$

The profile normal belonging to the parameter $u$ of the rack, meshes the rolling straight (line) of the rack at the point $x=\delta$. The u parameter point of the rack will roll into the main point after the value of $-\delta$. The complex equation of the connecting line can be expressed with the function of the rack:

$$
M=z_{1}(u)-\delta+I \cdot z_{2}(u)
$$




\section{Applications of the Theory}

In this chapter, three examples will be shown to illustrate the application of the presented method, by generating different geometry gears such as a cosine profile gear, the profile of the lobes of a Roots blower and also generating a general involute gear. These examples show that this analytical method can be easily used for very different problems, for designing conventional and non-conventional gears.

\subsection{Generating a Cosine Gear Profile}

Using the method herein, an example of generating a gear and its generating rack that has a cosine profile is shown. The advantages of this profile and a manufacturing method were described in [5]. A different and simpler method is given for the generating process.

The rack profile is described by the following equation:

$$
z=u+I \cdot\left(r+10 \cdot \sin \left(\frac{u}{5}\right)\right)
$$

In this example the radius of the pitch circle of the gear is $r=50 \mathrm{~mm}$. Substituting the values of $\mathrm{r}$, the number of teeth $(N)$ and the expression (15) into equation (2) provides the following formula:

$$
w=\left(u+\left(50+10 \cdot \sin \left(\frac{u}{5}\right)\right) \cdot I+50 \cdot \varphi\right) \cdot e^{(\varphi \cdot I)}
$$

The coordinate values for the abscissa and the ordinate of the real $\mathrm{R}(\mathrm{w})$ and imaginary $\mathrm{I}(\mathrm{w})$ part of $\mathrm{w}$ are assigned to variables as $X=R(w)$ and $Y=I(w)$.

The values of the coordinates are evaluated by partial derivation of $X$ and $Y$ according to $\mathrm{u}$ and $\varphi$ shown in (17):

$$
\begin{aligned}
& \frac{\partial}{\partial u} X=\cos (\varphi)-\sin \left(\frac{u}{5}+\varphi\right)-\sin \left(-\frac{u}{5}+\varphi\right) \\
& \frac{\partial}{\partial \varphi} X=-\sin (\varphi) u-50 \cdot \sin (\varphi) \cdot \varphi-5 \cdot \sin \left(\frac{u}{5}+\varphi\right)+5 \cdot \sin \left(-\frac{u}{5}+\varphi\right) \\
& \frac{\partial}{\partial u} Y=\cos \left(-\frac{u}{5}+\varphi\right)+\cos \left(\frac{u}{5}+\varphi\right)+\sin (\varphi) \\
& \frac{\partial}{\partial \varphi} Y=-5 \cdot \cos \left(-\frac{u}{5}+\varphi\right)+5 \cdot \cos \left(\frac{u}{5}+\varphi\right)+\cos (\varphi) u+50 \cdot \cos (\varphi) \cdot \varphi
\end{aligned}
$$

Using equation (3) a differential equation of the profile is defined that is made equal to zero (18): 
$\frac{\frac{\partial}{\partial \varphi} I(w(u, \varphi))}{\frac{\partial}{\partial \varphi} \operatorname{Re}(w(u, \varphi))}-\frac{\frac{\partial}{\partial u} I(w(u, \varphi))}{\frac{\partial}{\partial u} \operatorname{Re}(w(u, \varphi))}=0$

By expanding equation (18) the following equation is obtained:

$$
\begin{aligned}
& \frac{\cos \left(-\frac{u}{5}+\varphi\right)+\cos \left(\frac{u}{5}+\varphi\right)+\sin (\varphi)}{\cos (\varphi)-\sin \left(\frac{u}{5}+\varphi\right)-\sin \left(-\frac{u}{5}+\varphi\right)}- \\
& -\frac{-5 \cdot \cos \left(-\frac{u}{5}+\varphi\right)+5 \cdot \cos \left(\frac{u}{5}+\varphi\right)+\cos (\varphi) u+50 \cdot \cos (\varphi) \cdot \varphi}{-\sin (\varphi) u-50 \cdot \sin (\varphi) \cdot \varphi-5 \cdot \sin \left(\frac{u}{5}+\varphi\right)+5 \cdot \sin \left(-\frac{u}{5}+\varphi\right)}=0
\end{aligned}
$$

Equation (19) can be solved analytically. The closed form solution according to $\varphi$ can be given as:

$$
\varphi=-\frac{2 \cdot \cos \left(\frac{u}{5}\right) \cdot \sin \left(\frac{u}{5}\right)}{5}-\frac{u}{5}
$$

This solution can be further simplified using the following trigonometric identity:

$$
2 \cdot \cos \left(\frac{u}{5}\right) \cdot \sin \left(\frac{u}{5}\right)=\sin \left(\frac{2 u}{5}\right)
$$

The formula of the gear profile $(w)$ can be determined using the simplified formula of equation (21):

$$
\left.w=\left(\left(50+10 \cdot \sin \left(\frac{u}{5}\right)\right) I-10 \cdot \sin \left(\frac{2 \cdot u}{5}\right)\right) e^{\left(\left(\frac{\sin \left(\frac{2 \cdot u}{5}\right)}{5}-\frac{u}{50}\right)\right.} I\right)
$$

The contact curve of the cosine gears can be formulated using equation (14) as:

$$
M=-20 \cdot \cos \left(\frac{u}{5}\right) \cdot \sin \left(\frac{u}{5}\right)+\left(50+10 \cdot \sin \left(\frac{u}{5}\right)\right) I
$$

The generated gear, the contact and the enveloping rack are shown in Fig. 4. The teeth profile of the cosine rack is obtained from the calculation. The gear described in [8] has a cosine profile on the generated gear, however, the profile on the rack is a complicated curve which is difficult to manufacture accurately. The method described above is simpler because the rack profile is a cosine curve that is easier and cheaper to manufacture accurately. The profile of the generated gear teeth is better suited for a proper gear. 


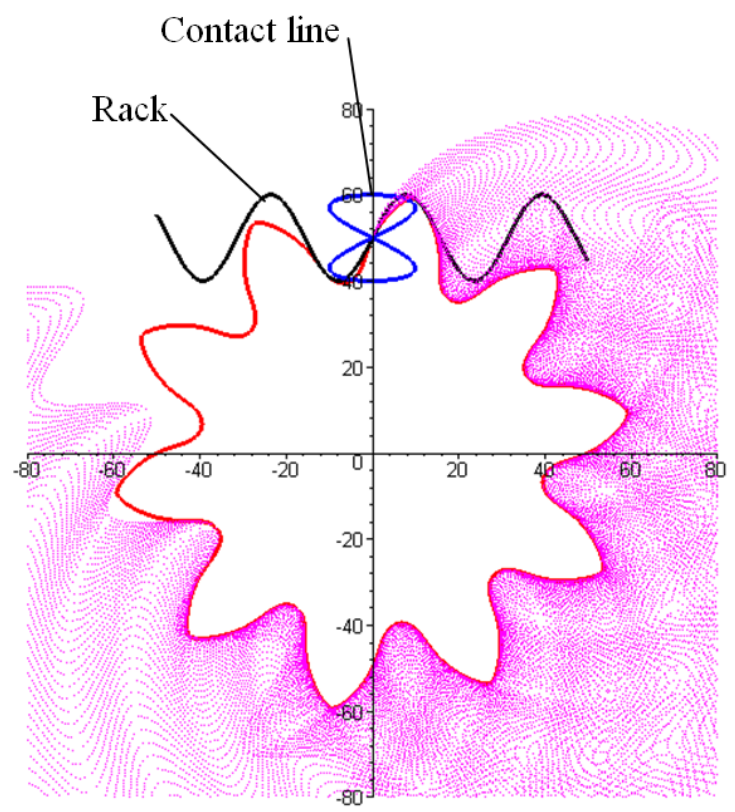

Figure 4

The full profile of the generated cosine gear enveloped by the rack and the contact line

\subsection{Generating the Profile of the Lobes of the Roots-Blower}

Another example of the presented theory is shown, where a rack profile is generated which can envelope the shape of the lobes of a Roots blower [6, 7]. The lobes of the blower consist of semicircles with the radius of $\rho=\frac{\pi}{2}$. The lobes connect tangentially to each order. In the case when the number of lobes is two, the radius of the acting pitch circle is $r=\frac{4 \rho}{\pi}$. The equation of the profiles applied to semicircles according to (1) is:

$$
p(u)= \begin{cases}-\sqrt{\rho^{2}-(u-\rho)^{2}} & \text { if } u<2 \cdot \rho \\ \sqrt{\rho^{2}-(u-3 \cdot \rho)^{2}} & \text { otherwise }\end{cases}
$$

Applying the Fourier series on the parametric formula (24) the following formulae can be generated (25):

$$
A_{j}=\frac{\int_{0}^{2 \pi} p(u) \cdot \sin (j \cdot u) d u}{\pi}
$$


$B_{j}=\frac{\int_{0}^{2 \pi} p(u) \cdot \cos (j \cdot u) d u}{\pi}$

Using these equations the formula of the rack profile can be given by the following expressions:

$$
\begin{aligned}
& z_{1}(u)=u \\
& z_{2}(u)=\frac{B_{0}}{2}+\left(\sum_{i=1}^{n}\left(A_{2 \cdot i-1} \cdot \sin ((2 i-1) \cdot u)+B_{2 i} \cdot \cos (2 i \cdot u)\right)\right)
\end{aligned}
$$

Using the formula (6) for (26):

$$
\begin{aligned}
& F_{1}=z_{1}(u) \\
& F_{2}=r+z_{2}(u)
\end{aligned}
$$

Using formulae (7) and (8) on (27) will generate the complete contour of the Roots-blower. The rolling of the rack can be presented by using equation (2). Fig. 5 shows the result of the whole motion of the generating process and the generated lobe. Fig. 6 shows the joined pair of lobes in its housing. The inside curvature of the housing has a radius of $R=r \cdot \sqrt{2}$. The movement of the rack will also generate additional profile elements which have a valid shape according to equation (8) but are never realized on a real work piece (see Fig. 6). These parts of the curve will be cut off by the generating tool. Nevertheless they are theoretically interesting because, they meet all the requirements expressed by differential equation (3).

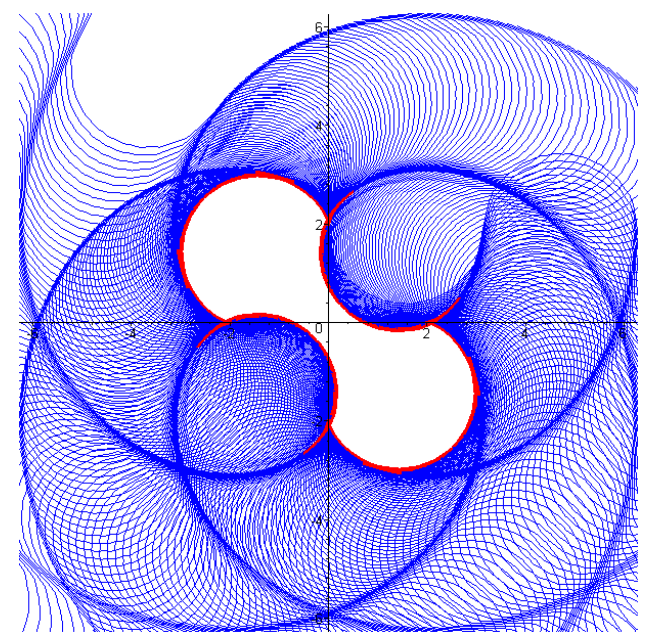

Figure 5

The generation of the profile of a Roots-blower lobe 


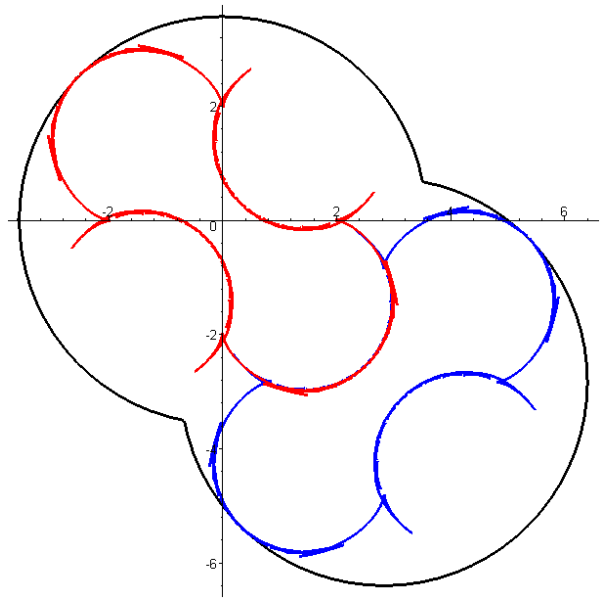

Figure 6

The profiles of the Roots-blowers according to the solution of the model.

\subsection{Generating the Profile of a Standard Involute Gear}

The last example, illustrates the design process of a general involute gear using our proposed method. The real differences between the mathematical point of view of gears and that of those realized at tooth generation, can be shown in more detail on involute gears using the method herein. The parametric equation of the rack (characterized by modulus $m=2$, basic angle $\alpha$, addendum high $h_{l}$ and dedendum $h_{2}$ the depth factors on the tool) is the following:

$$
p(u)= \begin{cases}\frac{u}{\tan (\alpha)} & \text { if } u<h_{1} \cdot \tan (\alpha) \\ h_{1} & \text { if } u<\pi-h_{1} \cdot \tan (\alpha) \\ \frac{\pi-u}{\tan (\alpha)} & \text { if } u<\pi+h_{2} \cdot \tan (\alpha) \\ -h_{2} & \text { if } u<2 \cdot \pi-h_{2} \cdot \tan (\alpha) \\ \frac{u-2 \cdot \pi}{\tan (\alpha)} & \text { otherwise }\end{cases}
$$

The Fourier series of the rack is generated according to equations (25) and (26). In general when the module of the gear is not two $(m \neq 2)$ and the profile displacement factor is not zero $(\xi \neq 0)$, in the Fourier series (26), the following equation holds:

$\hat{u}=\frac{2 u}{m}$ 
Carrying out the substitution (28) and (29) into (6) the formula for the rack profile is the following:

$$
\begin{aligned}
& F_{1}=z_{1}(\hat{u}) \\
& F_{2}=r+\frac{m}{2} \cdot z_{2}(\hat{u})+\xi \cdot m
\end{aligned}
$$

The process of translation and complex rotations is again carried out according to (7) and (8) to obtain the full gear profile. The results of the process can be seen in Fig. 7a a gear contour in the case of nine teeth $N=9, m=1.34, \alpha=20^{\circ}, h_{l}=1.25$, $h_{2}=1, \xi=0.2$ can be seen together with the generating tool movement. From Fig. 7 it is easy to see that profile generated according to formula (8) is wrong because, the number of teeth is too few, so undercuts will appear. Tooth sharpening can be partially seen on top of the generated teeth. In Fig. $7 \mathrm{~b}$ a variant of the previous solution can be seen, where no profile displacement $(\xi=0)$ was used.

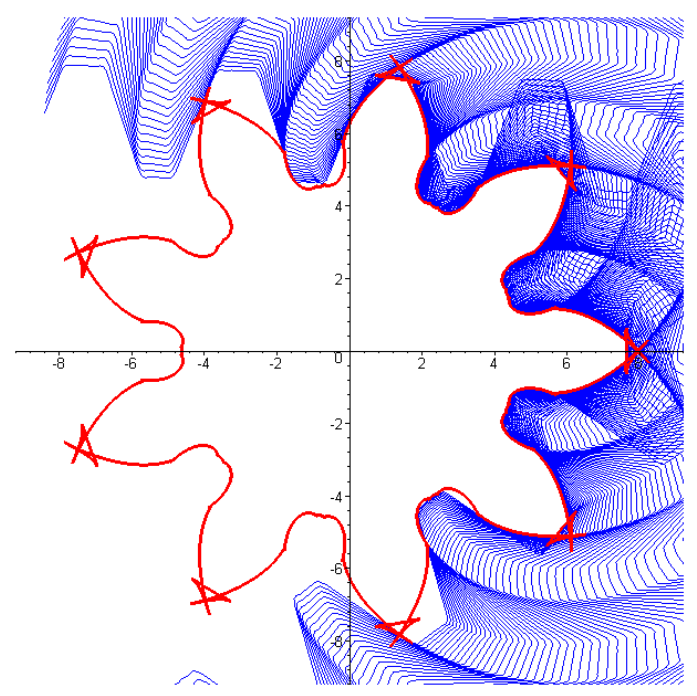

a) 


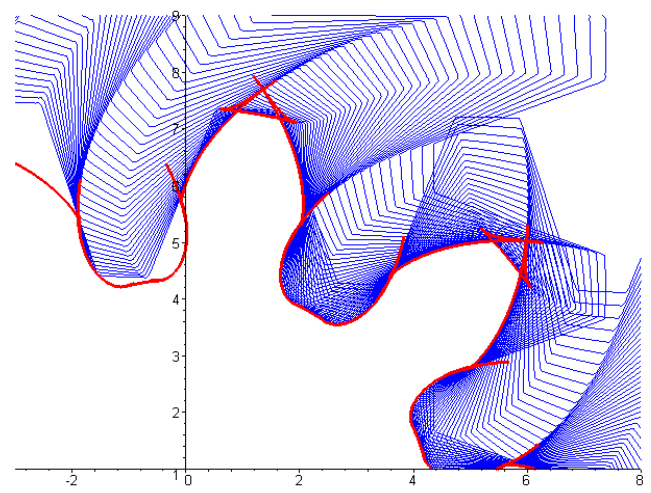

b)

Figure 7

Full (a) and partial (b) involute profiles with different values of undercut

\section{Conclusion and further remarks}

The method developed in this work is "manufacturing friendly". As it was seen, the non-conventional tooth profile and the rack (the tooth profile generating tool) are generated easily. This method uses complex algebra and complex functions that can be used for generating other general gear tooth profiles not described here. The demonstrated profiles were only shown here as examples, but an almost infinite variation of profiles can be generated with this method, as long as the tooth profile can be described by a suitable Fourier series.

\section{References}

[1] H.-CHR. GRAF SEHERR-THOSS: Die Entwicklung der Zahnradtechnik, Springer Verlag Berlin-Heidelberg-New York (1965)

[2] Faydor, L. Litvin, Alfonso Fuentes: Gear Geometry and Applied Theory, Cambridge University Press (2004)

[3] Laczik, B.: Design and Manufacturing of Non-Circular Gears by Given Transfer Function, ICT 2007 Conference, Miskolc, Hungary (2007) 101109

[4] Laczik, B.: Re-Discovery of the Non-Circular Gears, Manufacturing 2008 Conference, Budapest, Hungary (2008) 21-28

[5] Shanming Luo, Yue Wub, Jian Wang: The Generation Principle and Mathematical Model of a Novel Cosine Gear Drive, Mechanism and Machine Theory 43 (2008) 1543-1556 
[6] Shih-Hsi Tong, Daniel C. H. Yang: On the Generation of New Lobe Pumps for Higher Pumping Flowrate, Mechanism and Machine Theory 35 (2000) 997-1012

[7] Ligang Yao, Zhonghe Ye, Jian S. Dai, Haiyi Cai: Geometric Analysis and Tooth Profiling of a Three-Lobe Helical Rotor of the Roots blower, Journal of Materials Processing Technology 170 (2005) 259-267

[8] Laczik, B., Zentay, P., Horváth, R.: Modelling the Gear Generating Process with Complex Functions, $7^{\text {th }}$ International Conference on Mechanical Engineering, Budapest, Hungary (2010) 496-501 